\title{
Unconventional $p$-d Hybridization Interaction in PtGa Ultrathin Nanowires Boosts Oxygen Reduction Electrocatalysis
}

Lei Gao, ${ }^{\dagger}, \|$ Xingxing Li,,$\stackrel{+}{ }$ Zhaoyu Yao, ${ }^{\dagger}$ Huijuan Bai, ${ }^{\#}$ Yangfan Lu, ${ }^{\S}$ Chao Ma, ${ }^{\dagger}$ Shanfu Lu, Zhenmeng Peng, ${ }^{*, \pi}$ Jinlong Yang, ${ }^{\star}$ Anlian Pan,${ }^{\dagger}$ and Hongwen Huang ${ }^{*, \dagger, \S}$

College of Materials Science and Engineering, Hunan University, Changsha, Hunan 410082, P. R. China.

Hefei National Laboratory for Physical Sciences at the Microscale, Department of Chemical Physics, University of Science and Technology of China, Hefei, Anhui 230026, P. R. China.

\#Beijing Key Laboratory of Bio-inspired Energy Materials and Devices, School of Space and Environment, Beihang University, Beijing 100191, P. R. China.

§State Key Lab of Silicon Materials, School of Materials Science and Engineering, Zhejiang University, Hangzhou 310027, P. R. China.

IDepartment of Chemical and Biomolecular Engineering, University of Akron, Akron, Ohio 44325, United States.

"These authors contributed equally to this work.

Correspondence and requests for materials should be addressed to H.H. (email: huanghw@hnu.edu.cn) or Z.P. (zpeng@uakron.edu). 


\section{EXPERIMENTAL SECTION}

Chemicals. Platinum (II) acetylacetonate ( $\left.\mathrm{Pt}(\mathrm{acac})_{2}, 97 \%\right)$, gallium (III) acetylacetonate $\left(\mathrm{Ga}(\mathrm{acac})_{3}, 97 \%\right)$, oleylamine $(\mathrm{OAm},>90 \%)$, cetyltrimethylammonium bromide (CTAB, $\left.>99 \%\right)$, and Nafion (5wt. \%) were purchased from Sigma-Aldrich. Tungsten hexacarbonyl (W(CO) 97\%) was purchased from Alfa Aesar. Ascorbic Acid $\left(\mathrm{C}_{6} \mathrm{H}_{8} \mathrm{O}_{6}, \mathrm{AA}, 99 \%\right)$, ethanol $\left(\mathrm{CH}_{3} \mathrm{CH}_{2} \mathrm{OH}\right.$, analytical reagent, $>99.7 \%)$, isopropanol $\left(\mathrm{C}_{3} \mathrm{H}_{8} \mathrm{O}\right.$, analytical reagent, $\left.>99.7 \%\right)$ and perchloric acid $\left(\mathrm{HClO}_{4}\right.$, analytical reagent, $\left.70 \%-72 \%\right)$ were purchased from Sinopharm Chemical Reagent Co. Ltd. (Shanghai, China). The water $(18.2 \mathrm{M} \Omega / \mathrm{cm})$ used in all experiments was prepared by an ultra-pure purification system (Master-515Q, HHitech). All the chemicals were used without further purification.

Preparation of Pt nanowires. In a typical synthesis, $\mathrm{Pt}(\mathrm{acac})_{2}(10.0 \mathrm{mg}, 0.025 \mathrm{mmol})$, CTAB (90.0 mg, $0.25 \mathrm{mmol}$ ), and $4.0 \mathrm{~mL}$ oleylamine were added into a vial (volume: $30.0 \mathrm{~mL}$ ). After ultrasonication for $30 \mathrm{~min}, \mathrm{~W}(\mathrm{CO}) 6(15.0 \mathrm{mg}, 0.041 \mathrm{mmol})$ was added into the mixture solution and sealed. The mixture solution was proceeded at $160{ }^{\circ} \mathrm{C}$ for 2 hours to obtain $\mathrm{Pt}$ nanowires (NWs), and cooled to room temperature. The resulting colloidal materials were collected by centrifugation twice with a hexane/ethanol mixture.

Preparation of PtGa nanowires. For the synthesis of PtGa nanowires, the Pt nanowires were used as a seed. In brief, Pt NWs (3.0 mg, $0.015 \mathrm{mmol}), \mathrm{Ga}(\mathrm{acac})_{3}(36.7 \mathrm{mg}, 0.1 \mathrm{mmol})$, and AA (100.0 mg, $0.57 \mathrm{mmol})$ were added sequentially into $30.0 \mathrm{~mL}$ vial containing $4 \mathrm{~mL}$ OAm. After the vial had been capped, the solution was mixed under ultrasonication for $1 \mathrm{~h}$. The mixture solution was proceeded at $170{ }^{\circ} \mathrm{C}$ for 24 hours in an oil bath to obtain PtGa NWs, and cooled to room temperature. The resulting colloidal products were collected by centrifugation three times with a hexane/ethanol mixture.

Electrochemical measurements for ORR. Prior to the electrochemical measurements, the carbon-supported NWs as catalysts were prepared. Typically, a chloroform solution $(8.0 \mathrm{~mL})$ containing $4 \mathrm{mg}$ of the PtGa or Pt NWs was dropwise added to an ethanol solution containing 16 $\mathrm{mg}$ of carbon support (Vulcan XC-72) under vigorous stirring for $30 \mathrm{~min}$. The obtained carbon-supported NWs were then collected and washed twice with hexanes by centrifugation (10,000 rpm). Subsequently, the $\mathrm{Pt}_{\mathrm{x}} \mathrm{Ga} \mathrm{NWs} / \mathrm{C}$ and $\mathrm{Pt} \mathrm{NWs} / \mathrm{C}$ catalysts were dispersed in acetic acid and heated at $70{ }^{\circ} \mathrm{C}$ for $12 \mathrm{~h}$ to remove the organic surfactants and amorphous $\mathrm{GaO}_{\mathrm{x}}$ on the surface of PtGa NWs. The final catalysts were collected by centrifugation, and washed with 
ethanol for five times. After being dried, the catalysts were re-dispersed in the mixture containing $0.5 \mathrm{~mL}$ isopropanol, $0.495 \mathrm{~mL}$ ethanol and $0.005 \mathrm{~mL}$ Nafion (5 wt. \%) to form the well-mixed catalyst ink by sonicating for $1 \mathrm{~h}$. For the commercial Pt/C catalysts (20 wt. \% loading, Johnson Matthey), the suspension solution was prepared with a catalyst concentration of $0.4 \mathrm{mg}_{\mathrm{Pt}} / \mathrm{mL}$. Finally, $10 \mu \mathrm{L}$ of prepared catalyst ink was dropped onto the glassy carbon rotating disk electrode (GC, RDE with geometric area of $0.196 \mathrm{~cm}^{2}$ ) with the loading amount of Pt at 2.5 $\mu \mathrm{g}, 2.5 \mu \mathrm{g}$ and $4.0 \mu \mathrm{g}$ for $\mathrm{Pt}_{\mathrm{x}} \mathrm{Ga} \mathrm{NWs} / \mathrm{C}, \mathrm{Pt} \mathrm{NWs} / \mathrm{C}$ and commercial Pt/C catalysts, respectively (based on ICP-AES).

Electrochemical tests were conducted on a CHI760e electrochemical work station (Chenhua Instrument, China) in a three-electrode cell. A glassy carbon Rotating Disk Electrode (RDE, diameter: $5 \mathrm{~mm}$ ) was used as the working electrode, the $\mathrm{Ag} / \mathrm{AgCl}(3 \mathrm{M} \mathrm{KCl})$ electrode was used as a reference electrode, and a platinum wire was used as a counter electrode. All potentials were converted to the reversible hydrogen electrode reference. The cyclic voltammograms (CVs) were tested in a $\mathrm{N}_{2}$-saturated $0.1 \mathrm{M} \mathrm{HClO}_{4}$ electrolyte with a sweep rate of $50 \mathrm{mV} \mathrm{s}$. The electrochemical active surface areas (ECSAs) ECSAs were calculated by integrating the hydrogen adsorption/desorption charge area between 0.05 and $0.38 \mathrm{~V}_{\mathrm{RHE}}$ from the CVs. The charge density for the adsorption of one monolayer hydrogen on $\mathrm{Pt}\left(\mathrm{Q}_{\mathrm{H}}\right)$ was assumed to be 210 $\mu \mathrm{C} \mathrm{cm}{ }^{-2}$. For CO stripping measurements, CO gas $(99.99 \%)$ was fed into the electrolyte with holding a potential of $0.1 \mathrm{~V}_{\mathrm{RHE}}$ for $30 \mathrm{~min}$. Then, $\mathrm{N}_{2}$ gas was purged to remove $\mathrm{CO}$ from the electrolyte, and the CO stripping curves were recorded between 0.05 and $1.05 \mathrm{~V}_{\mathrm{RHE}}$ with a sweep rate of $50 \mathrm{mV} \mathrm{s}^{-1}$. The charge density for the monolayer adsorption of $\mathrm{CO}$ on Pt surfaces was assumed as $420 \mu \mathrm{C} \mathrm{cm}^{-2}$. The ORR polarization curves were measured in an $\mathrm{O}_{2}$-saturated $0.1 \mathrm{M}$ $\mathrm{HClO}_{4}$ solution between 0.05 and $1.05 \mathrm{~V}_{\mathrm{RHE}}$ using a sweep rate of $10 \mathrm{mV} \mathrm{s}^{-1}$ at rotation rate of $1600 \mathrm{rpm}$. During the accelerated durability tests (ADTs), each catalyst was recorded via cyclic sweeps between 0.6 and $1.1 \mathrm{~V}_{\mathrm{RHE}}$ in a $0.1 \mathrm{M} \mathrm{HClO}_{4}$ electrolyte at a sweep rate of $100 \mathrm{mV} \mathrm{s}^{-1}$ for 30,000 cycles.

Membrane electrode assembly (MEA) fabrication and single-cell test. The catalysts activity was evaluated under PEMFC operating conditions. Specifically, $\mathrm{Pt}_{4.31} \mathrm{Ga} \mathrm{NWs} / \mathrm{C}$, commercial $\mathrm{Pt} / \mathrm{C}$ were employed as the cathode catalysts and $\mathrm{Pt} / \mathrm{C}$ (20 wt. \% loading, Johnson Matthey) was used for the anode. Catalysts $(4.5 \mathrm{mg})$ were mixed with isopropanol $(1 \mathrm{~mL})$, deionized water $(300 \mu \mathrm{L})$, and Nafion alchol solution $(48 \mu \mathrm{L})$ to prepare the ink, which 
contained the Nafion ionomer-to-catalyst weight ratio of 0.55 . The active area of electrodes was $4 \mathrm{~cm}^{2}$. The formed catalyst ink was prepared by ultrasonicating for $2 \mathrm{~h}$ and sprayed directly onto a Nafion 211 membrane (DuPont). The Pt loading at the cathode was $0.12 \mathrm{mg}_{\mathrm{Pt}} \mathrm{cm}^{-2}$ for PtGa NWs and $0.14 \mathrm{mg}_{\mathrm{Pt}} \mathrm{cm}^{-2}$ for commercial $\mathrm{Pt} / \mathrm{C}$, respectively. As for the anode, $\mathrm{Pt} / \mathrm{C}$ was used with a loading of $0.15 \mathrm{mg}_{\mathrm{Pt}} \mathrm{cm}^{-2}$. The $\mathrm{H}_{2}-\mathrm{O}_{2}$ fuel cells were tested (Greenlight $\mathrm{G} 20$ test system and Princeton $\mathrm{P} 4000+$ electrochemical workstation) under galvanic mode using humidified $\mathrm{H}_{2}$ and $\mathrm{O}_{2}$ gases. The cell temperature was set to $70{ }^{\circ} \mathrm{C}$, and the flow rate of both $\mathrm{H}_{2}$ (without backpressure $)$ and $\mathrm{O}_{2}$ (0.5 bar of backpressure) gases was $200 \mathrm{~mL} / \mathrm{min}$ and $300 \mathrm{~mL} / \mathrm{min}$.

DFT calculations. To simulate the Pt NWs and PtGa alloy NWs, a seven-layer 2x2 Pt (111) and 1x1 Pt3Ga (111) slab is constructed, respectively (Figure S20). Furthermore, according to the experiment, the outmost layer of Pt3Ga (111) slab is replaced by pure Pt-skin monolayers. To analyze the chemical bonding interaction between Ga and Pt atoms, the electron localization function is calculated, which is a semiquantitative index of electron localization: a value of 0 means completely delocalization, while a value of 1 means completely localization. ${ }^{1,2}$ To study oxygen adsorption, only the adsorption on fcc hollow site is considered and the adsorption energy is calculated as $\mathrm{E}_{\mathrm{O}}=\mathrm{E}_{\text {slab-2O }}-\mathrm{E}_{\text {slab }}-\mathrm{E}_{\mathrm{O} 2}$ where $\mathrm{E}_{\text {slab-2O }}$ is the total energy of slab with two $\mathrm{O}$ atoms adsorbed on the top and bottom Pt layers respectively, $\mathrm{E}_{\text {slab }}$ is the total energy of pure slab, and $\mathrm{E}_{\mathrm{O} 2}$ is the total energy of oxygen molecule. To understand the different stability of Pt NWs and PtGa bimetallic NWs, the vacancy formation energy of outmost Pt atom is computed by $\mathrm{E}_{\mathrm{V}(\mathrm{Pt})}=\mathrm{E}_{\text {slab-V(Pt) }}+\mu \mathrm{Pt}-\mathrm{E}_{\text {slab }}$, where $\mathrm{E}_{\text {slab-V(Pt) }}$ and $\mu \mathrm{Pt}$ are the total energy of slabs with one Pt vacancy and chemical potential of Pt atom (set to be the energy of face-centered cubic Pt crystal), respectively. In this case, the 4x4 Pt (111) and 2x2 Pt3Ga (111) slabs are used.

All calculations are carried out within the Perdew-Burke-Ernzerhof generalized gradient approximation $(\mathrm{GGA})^{3}$ with D3 type van der Waals interaction (vdW) correction ${ }^{4,5}$ implemented in Vienna ab initio simulation package (VASP). ${ }^{6}$ The projector augmented wave (PAW) potential $^{7}$ and the plane-wave cut-off energy of $520 \mathrm{eV}$ are used. Monkhorst-Pack k-point meshes of $9 \times 9$ and $5 \times 5$ are employed when studying $\mathrm{O}$ adsorption and $\mathrm{Pt}$ vacancy formation, respectively. Adjacent slabs are separated by a vacuum space of $15 \AA$ along the z-direction to eliminate their interactions. During the simulations, both the lattice constants and positions of all atoms are fully relaxed until the force is less than $0.01 \mathrm{eV} / \AA$. The criterion for the total energy is set as $1 \times 10^{-6} \mathrm{eV}$. 
Characterization techniques. The crystalline phases of all samples were evaluated by X-ray diffraction (XRD, Rigaku Miniflex-600) patterns with a $\mathrm{Cu} K \alpha$ radiation $(\lambda=0.15406 \mathrm{~nm}$, $40 \mathrm{kV}$ ). TEM images were obtained using a JEM-2100plus electron microscope. HAADF-STEM images and elemental mapping results were recorded on a JEOL ARM-200F microscope with spherical aberration corrector operating at $200 \mathrm{kV}$. Elemental analysis of Pt and $\mathrm{Ga}$ were quantitatively conducted by ICP-AES with a SPECTRO BLUE SOP. The valence states of Pt and Ga were performed by X-ray photoelectron spectroscopy (XPS) using an Escalab 250Xi equipped with an $\mathrm{Al} \mathrm{Ka}(1486.6 \mathrm{eV})$ excitation source. All the spectra collected were corrected using a Shirley background. Compositions were determined by considering the atomic sensitivity factors. Centre of gravity calculation for the valence band spectra is conducted as $\frac{\int N(\varepsilon) \varepsilon d \varepsilon}{\int N(\varepsilon) d \varepsilon}$ in the range of $0 \sim-9.0 \mathrm{eV}$, where $N(\varepsilon)$ is the density of states. 

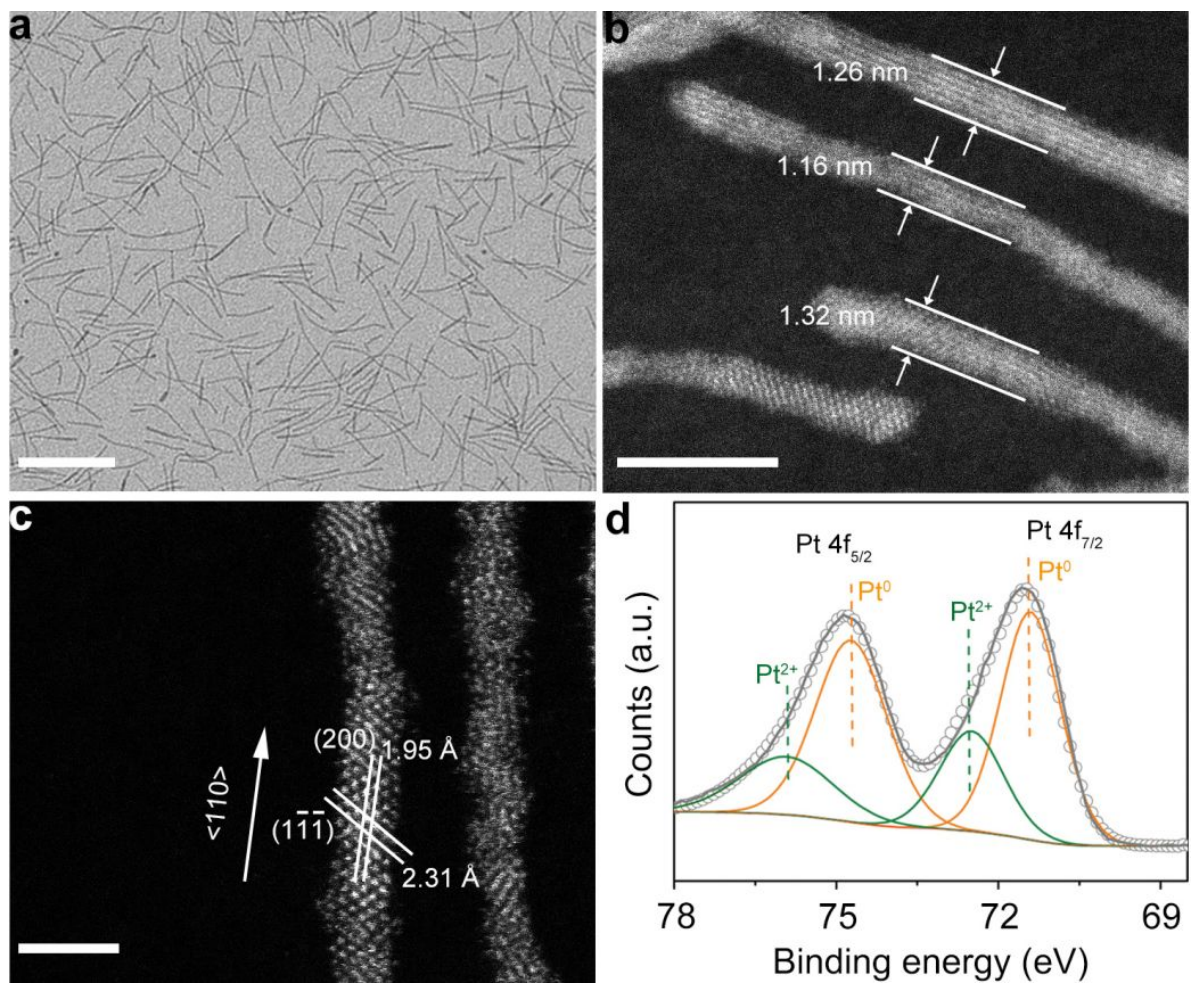

Supplementary Figure S1. Structural characterizations for Pt NWs. (a) low-magnification TEM image. Scale bar, $50 \mathrm{~nm}$. (b) HAADF-STEM image. Scale bar, $5 \mathrm{~nm}$. (c) Atomic-resolution HAADF-STEM image. Scale bars, $2 \mathrm{~nm}$. (d) High-resolution XPS spectrum of Pt 4f. 

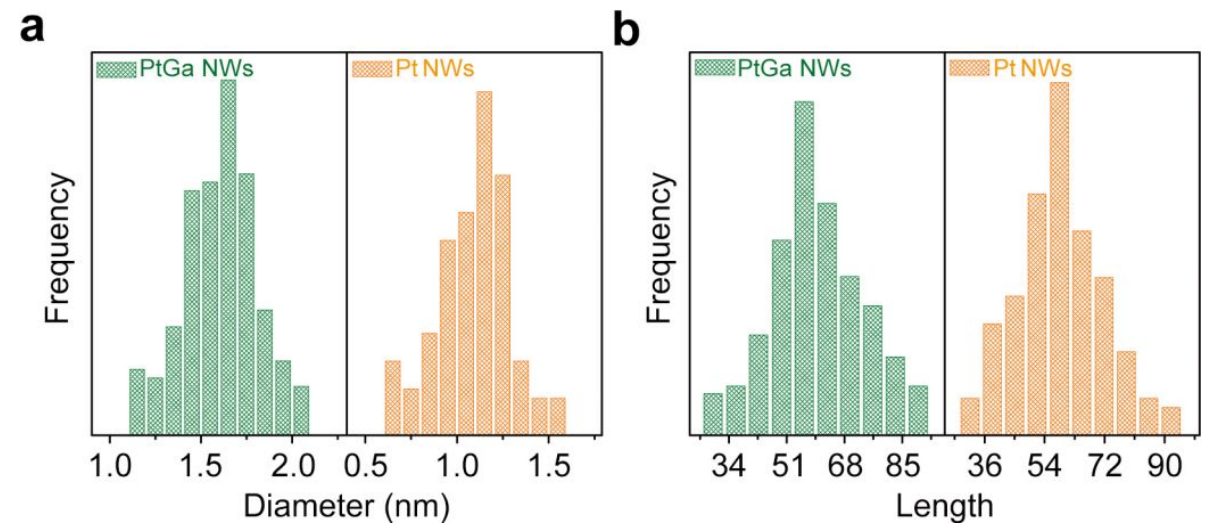

Supplementary Figure S2. Histogram of diameter and length distributions for the PtGa and Pt NWs. 

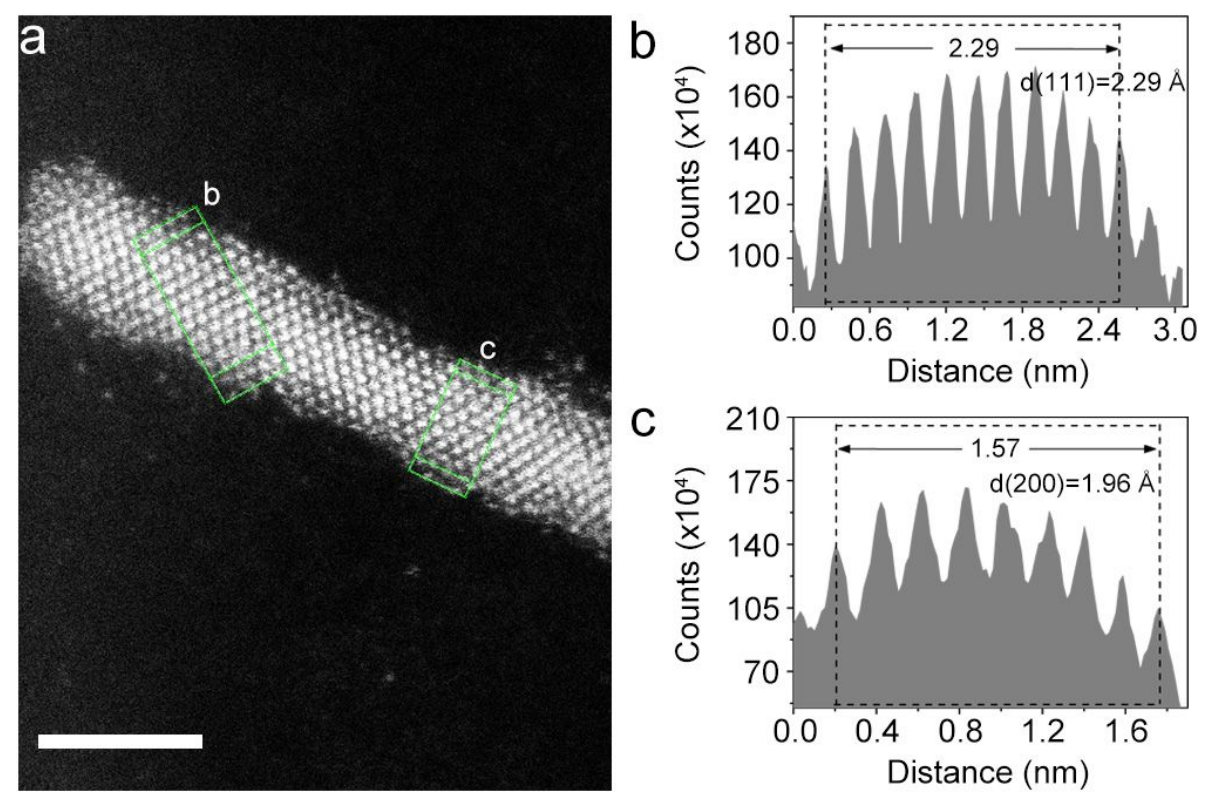

C

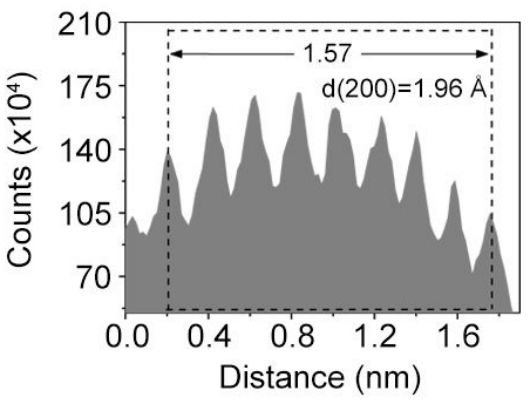

Supplementary Figure S3. (a) Atomic-resolution HAADF-STEM image of the PtGa NWs. Scale bar, $2 \mathrm{~nm}$. (b and c) Intensity profiles recorded from the area indicated by the rectangular box in panel (a). 


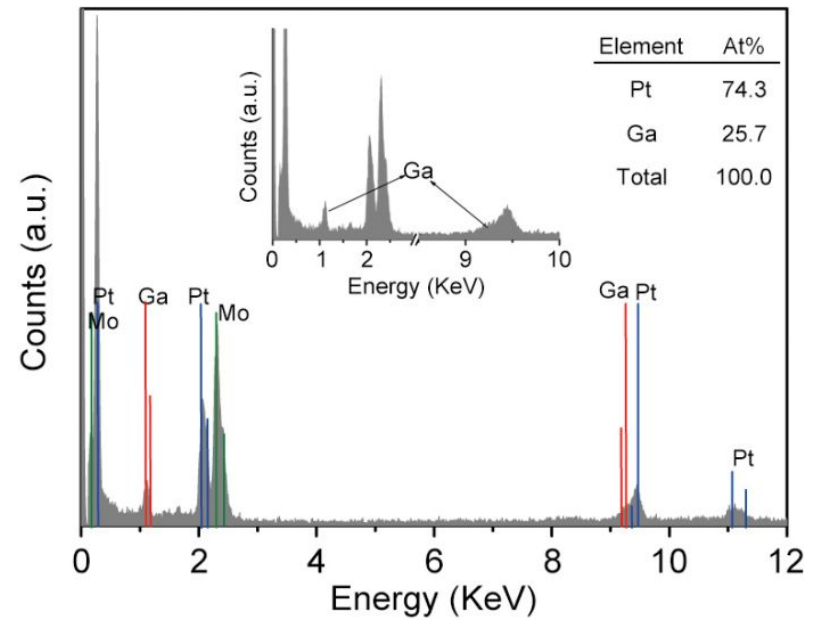

Supplementary Figure S4. TEM-EDS spectrum of PtGa NWs. 


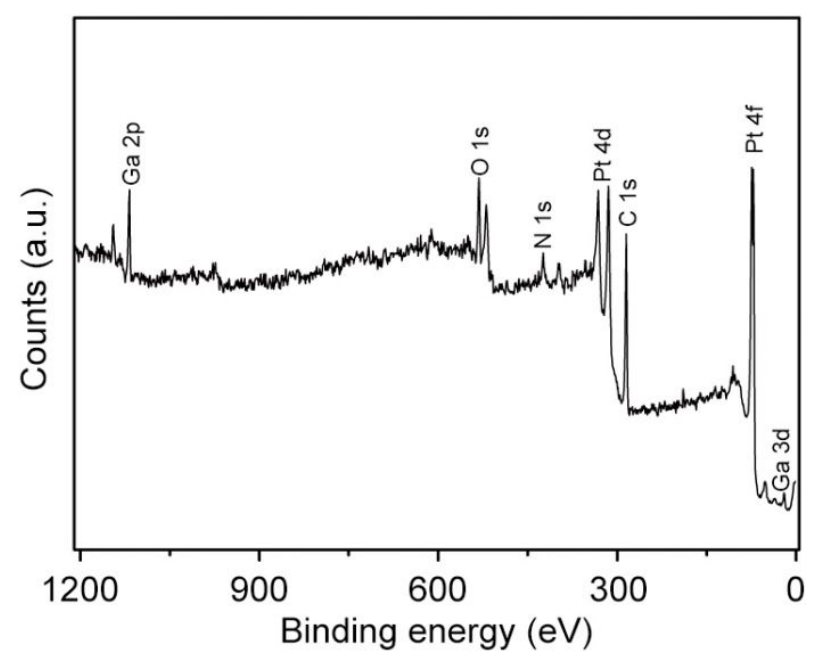

Supplementary Figure S5. The XPS survey spectrum of the PtGa NWs. 

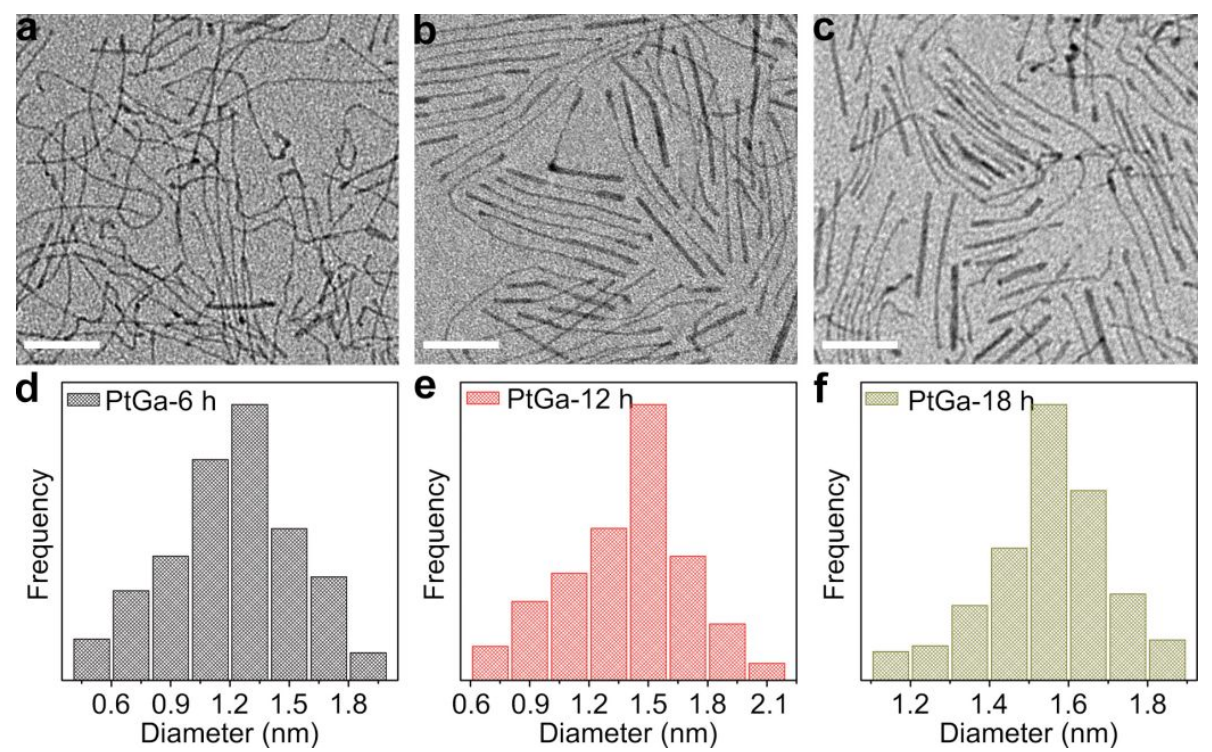

Supplementary Figure S6. TEM images (a-c) and histograms of diameter distributions (d-f) for the PtGa NWs at different reaction stages. (a, d) 6 h; (b, e) 12 h; (c, f) 18 h. Scale bars, $20 \mathrm{~nm}$. 


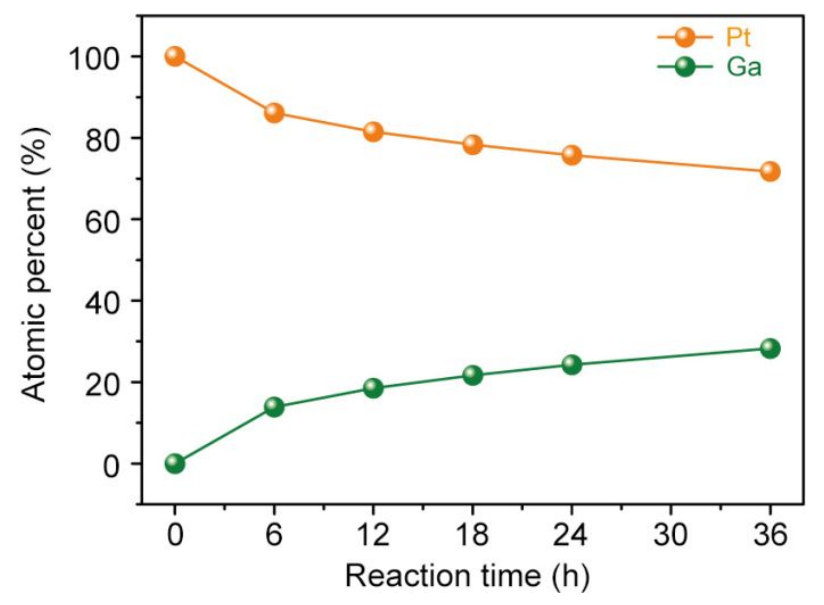

Supplementary Figure S7. The plot showing the time-dependent compositional evolution for the formation of PtGa NWs. 


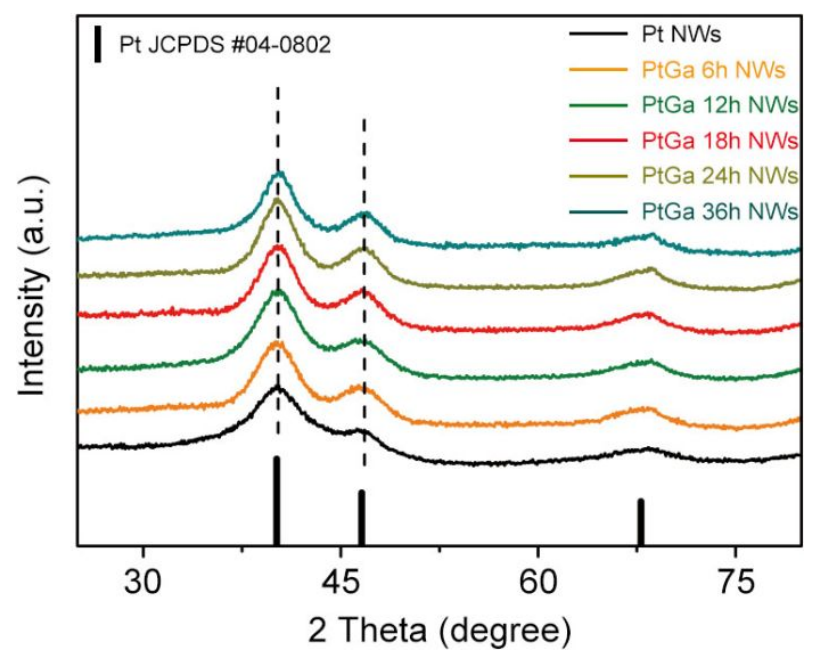

Supplementary Figure S8. PXRD patterns of Pt NWs and PtGa NWs at different reaction stages. 

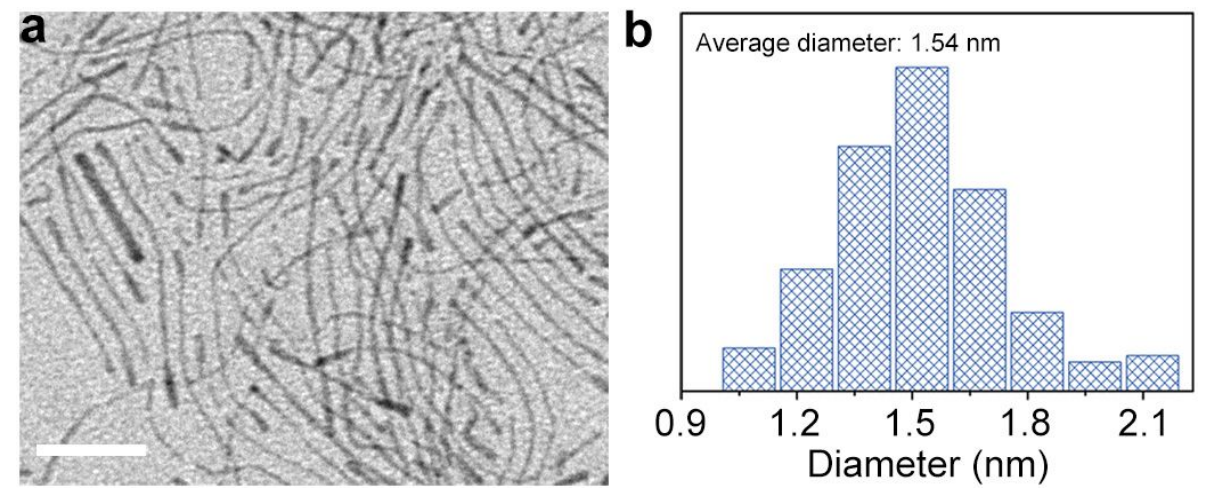

Supplementary Figure S9. TEM image (a) and histogram of diameter distribution (b) for the PtGa NWs obtained using the standard procedure except for the use of glucose to replace the ascorbic acid as the reductant. Scale bar, $20 \mathrm{~nm}$. 


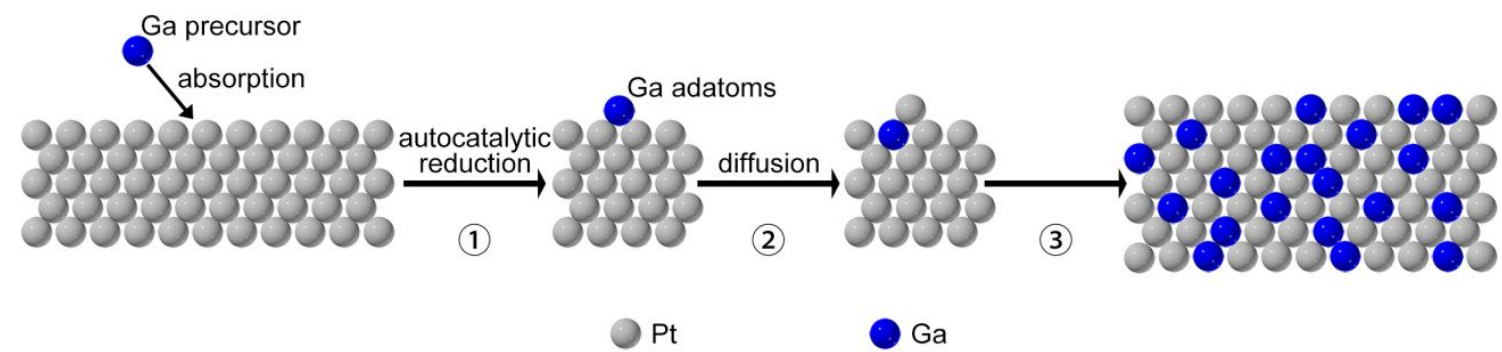

Supplementary Figure S10. Schematic illustration showing the formation mechanism of PtGa NWs at the atomic level. 


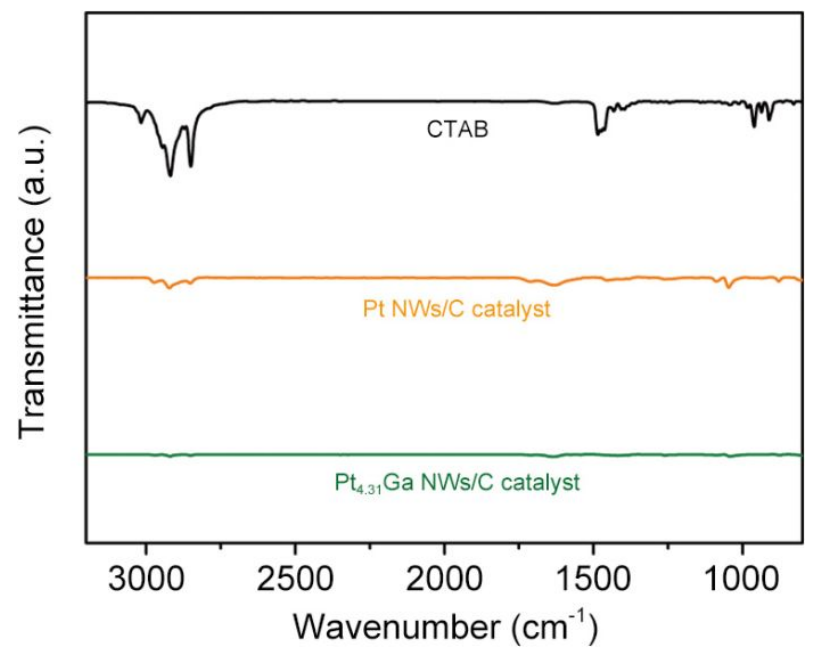

Supplementary Figure S11. FT-IR spectra recorded from CTAB, Pt NWs/C, $\mathrm{Pt}_{4.31} \mathrm{Ga} \mathrm{NWs}_{\mathrm{s}} \mathrm{C}$ catalysts, respectively. 


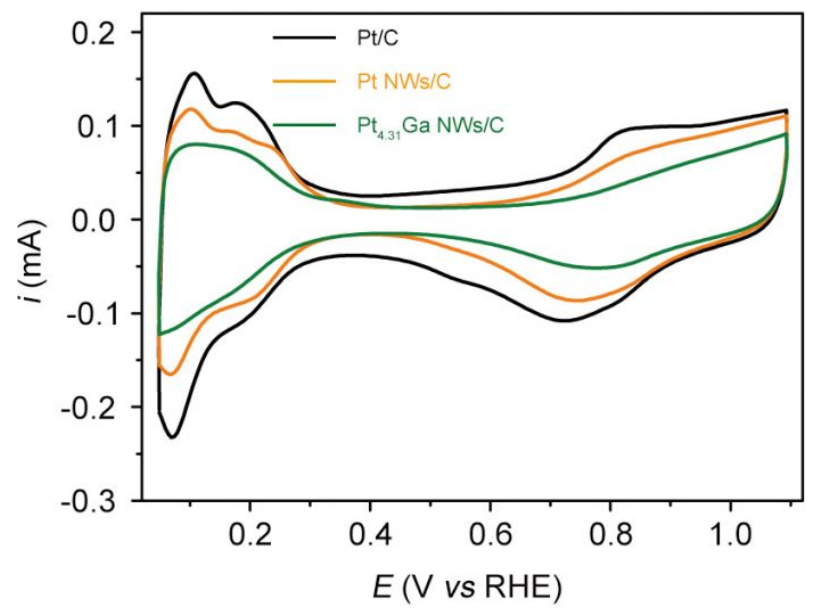

Supplementary Figure S12. CVs recorded at room temperature in $\mathrm{N}_{2}$-saturated $0.1 \mathrm{M} \mathrm{HClO}_{4}$ solutions at a sweep rate of $50 \mathrm{mV} \mathrm{s}^{-1}$. 

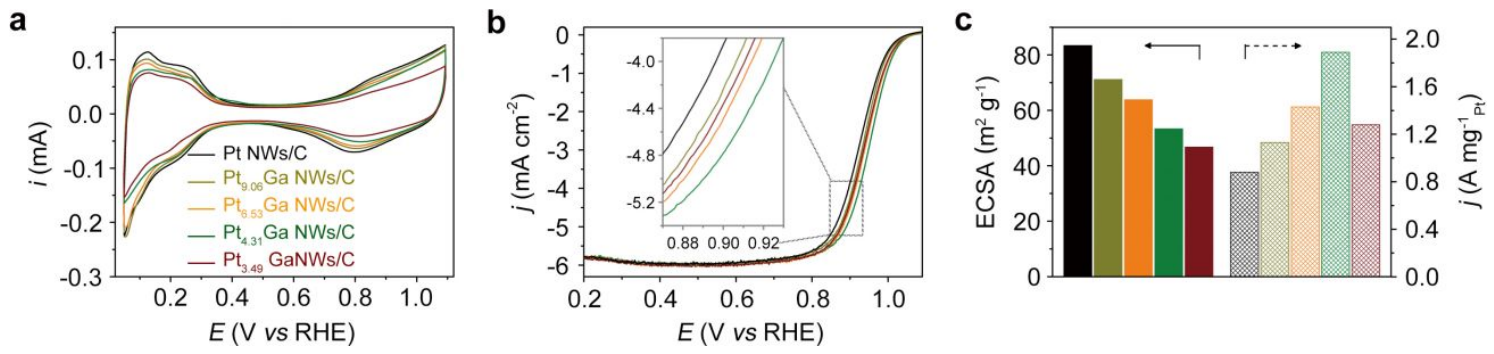

Supplementary Figure S13. Electrochemical activity of the catalysts. (a) CV curves, (b) ORR polarization curves, and (c) ECSA and mass activities of different catalysts. The color scheme in panel (a) applies to all other panels. 


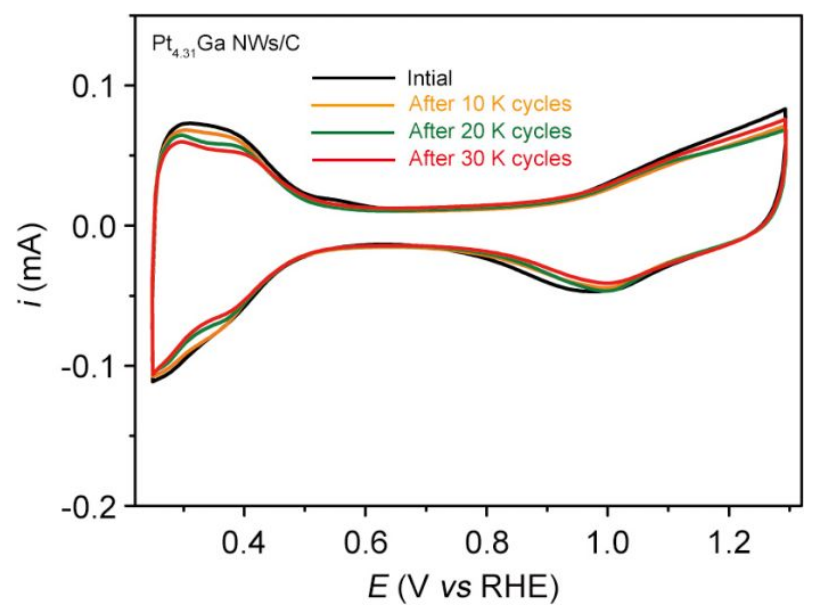

Supplementary Figure S14. CV curves of $\mathrm{Pt}_{4.31} \mathrm{Ga} \mathrm{NWs} / \mathrm{C}$ before and after different ADT cycles. 

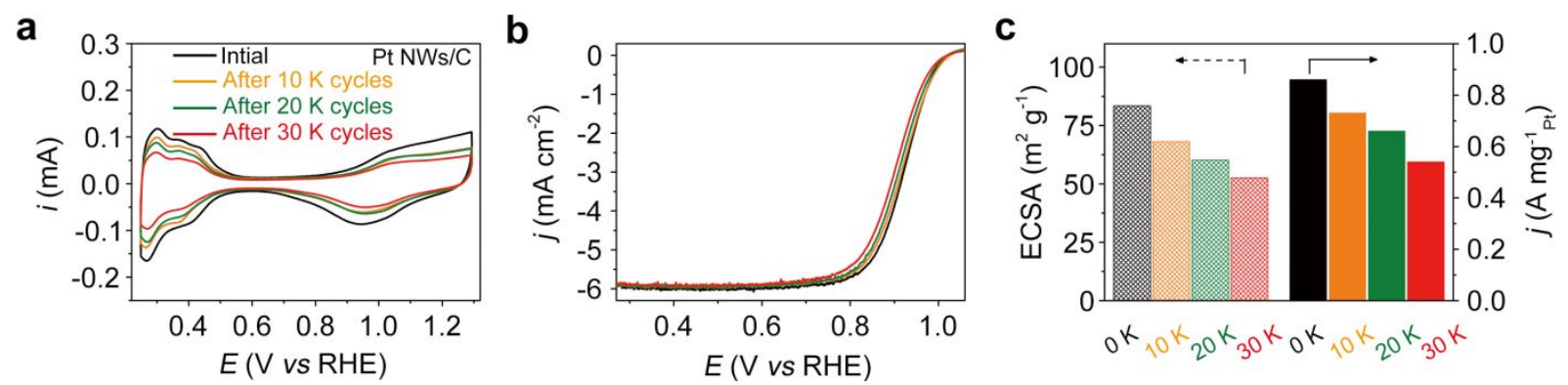

Supplementary Figure S15. Long-term durability test of the Pt NWs/C. (a) CV curves and (b) ORR polarization curves of $\mathrm{Pt} \mathrm{NWs} / \mathrm{C}$ before and after different ADT cycles. (c) The changes in ECSA and mass activities of Pt NWs/C before and after different ADT cycles. 

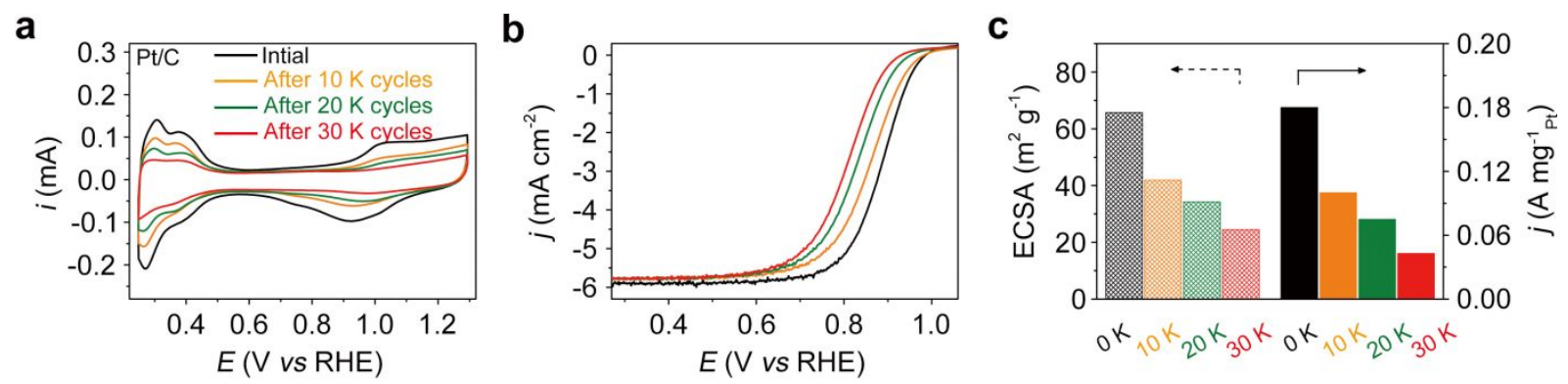

Supplementary Figure S16. Long-term durability test of the Pt/C. (a) CV curves and (b) ORR polarization curves of $\mathrm{Pt} / \mathrm{C}$ before and after different ADT cycles. (c) The changes in ECSA and mass activities of $\mathrm{Pt} / \mathrm{C}$ before and after different ADT cycles. 

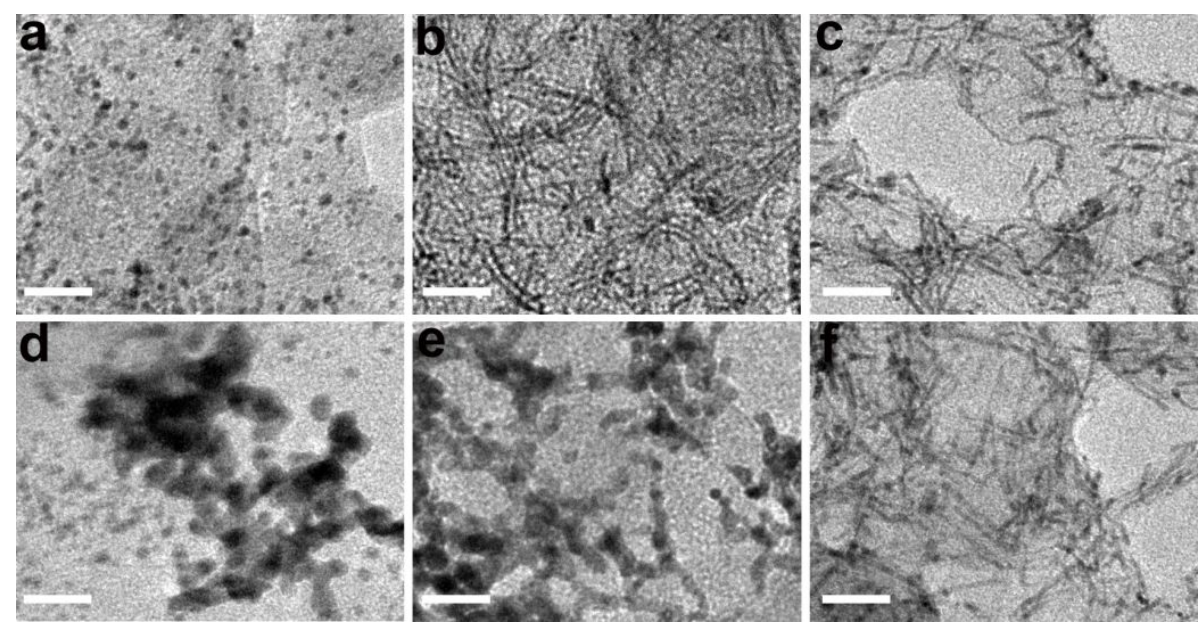

Supplementary Figure S17. (a-c) The TEM images of commercial Pt/C (a), $\mathrm{Pt} \mathrm{NWs/C} \mathrm{(b),} \mathrm{and}$ $\mathrm{Pt}_{4.31} \mathrm{Ga} \mathrm{NWs} / \mathrm{C}$ (c) catalysts before ADTs. (d-e) The TEM images of commercial Pt/C (a), Pt $\mathrm{NWs} / \mathrm{C}(\mathbf{b})$, and $\mathrm{Pt}_{4.31} \mathrm{Ga} \mathrm{NWs} / \mathrm{C}$ (c) catalysts after 30,000 cycles of ADTs. Scale bars, $20 \mathrm{~nm}$. 
a

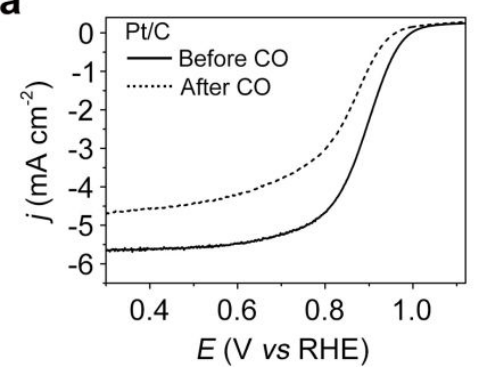

b

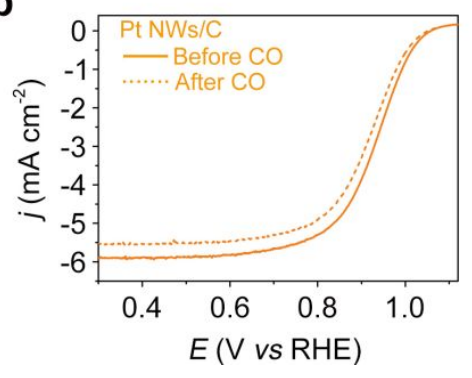

C

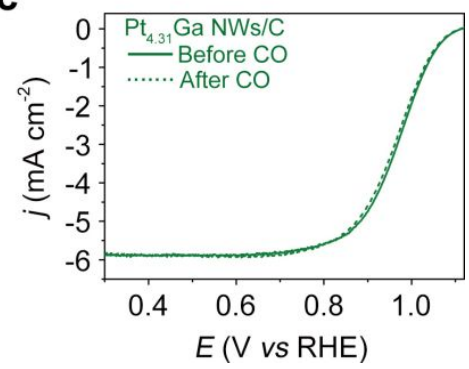

Supplementary Figure S18. ORR polarization curves of $\mathrm{Pt} / \mathrm{C}(\mathbf{a}), \mathrm{Pt} \mathrm{NWs} / \mathrm{C}(\mathbf{b})$, and $\mathrm{Pt}_{4.31} \mathrm{Ga}$ NWs/C (c) catalysts before and after CO-tolerance measurements. 

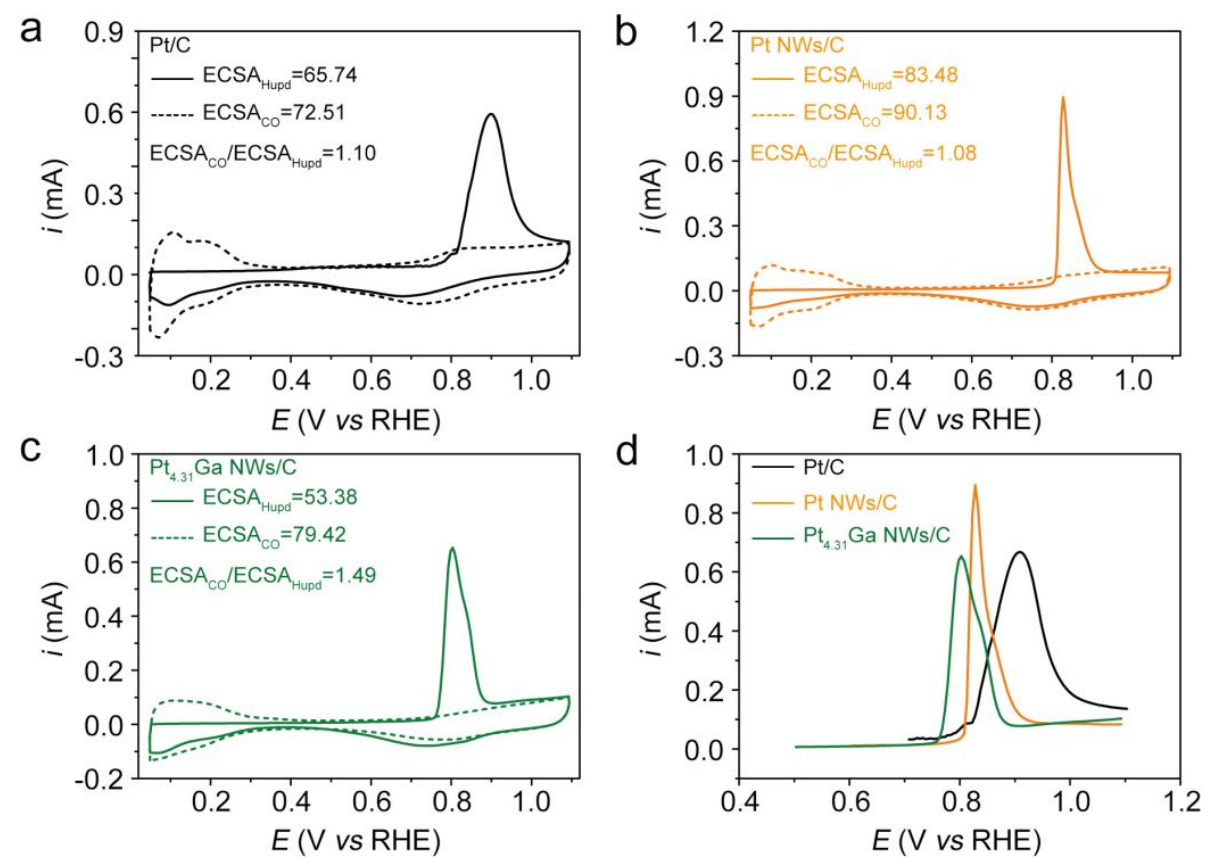

Supplementary Figure S19. CVs in N2-saturated electrolyte (dotted lines) and CO stripping curves (solid lines) to estimate the ECSAs for commercial Pt/C (a), Pt NWs/C (b), and $\mathrm{Pt}_{4.31} \mathrm{Ga}$ $\mathrm{NWs} / \mathrm{C}$ (c). (d) CO-stripping curves with $\mathrm{Pt}_{4.31} \mathrm{Ga} \mathrm{NWs} / \mathrm{C}$, Pt NWs/C, and commercial Pt/C catalysts carried out in $\mathrm{CO}$-saturated $0.1 \mathrm{M} \mathrm{HClO}_{4}$ electrolyte at room temperature with a scan rate of $50 \mathrm{mV} \mathrm{s}^{-1}$. 

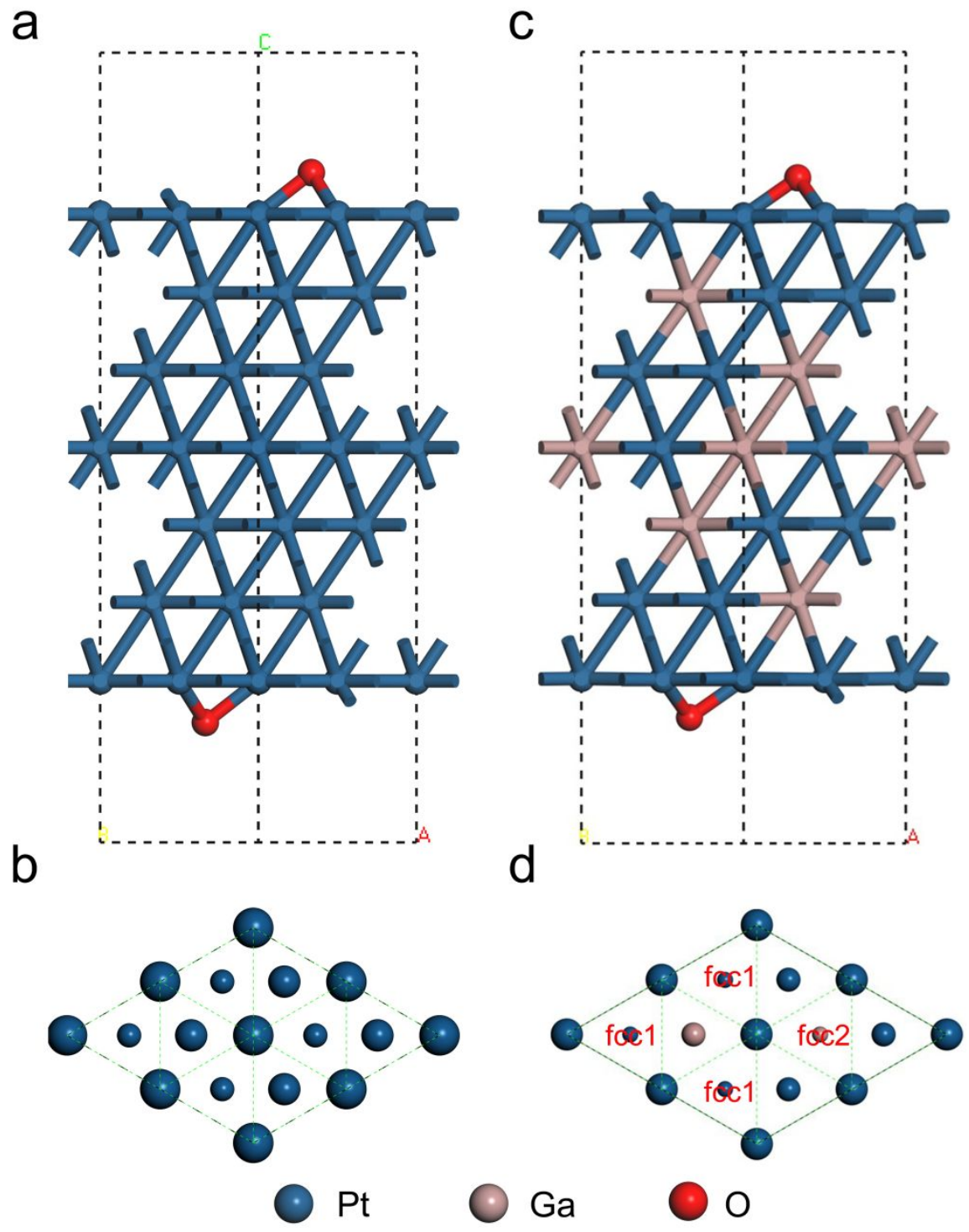

Supplementary Figure S20. Theoretical model structures of $\mathrm{Pt} \mathrm{NWs}(\mathbf{a}, \mathbf{b})$ and $\mathrm{Pt}_{4.31} \mathrm{Ga} \mathrm{NWs}$ $(\mathbf{c}, \mathbf{d})$. In panels $(\mathbf{b}, \mathbf{d})$, fcc sites indicate the hollow sites for possible adsorption of oxygen atoms. Noted that the fcc1 is the active site and fcc 2 is an inactive site. The big, medium and small sizes of balls indicate the topmost, the second and the third atomic layers, respectively. 


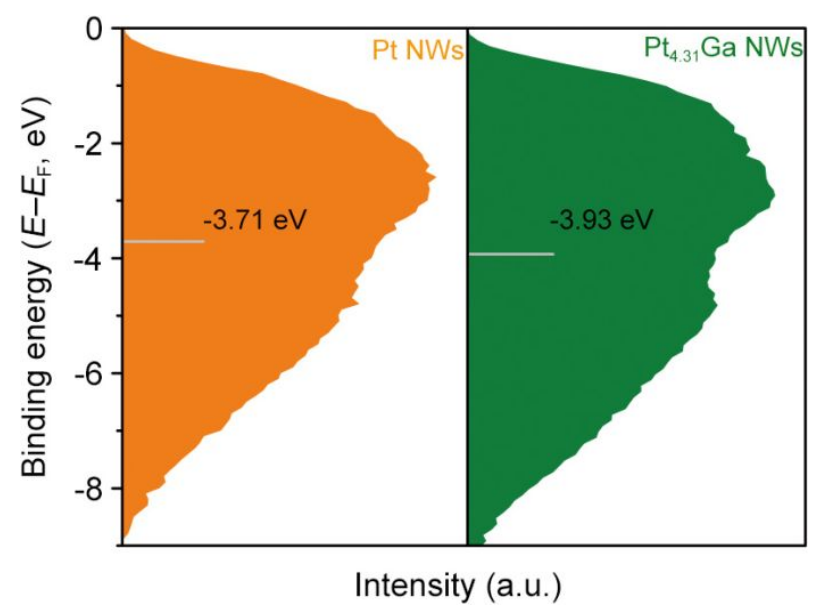

Supplementary Figure S21. Surface valence band photoemission spectra of Pt NWs and $\mathrm{Pt}_{4.31} \mathrm{Ga}$ NWs. All the spectra are background corrected. The white bars indicate its center of gravity. For comparison, the upper limit of integration is fixed to $-9.0 \mathrm{eV}$ in binding energy. 
Supplementary Table S1. Performance of $\mathrm{Pt}_{4.31} \mathrm{Ga} \mathrm{NWs} / \mathrm{C}$ catalyst in this work and several representative results with high performance from recent published works.

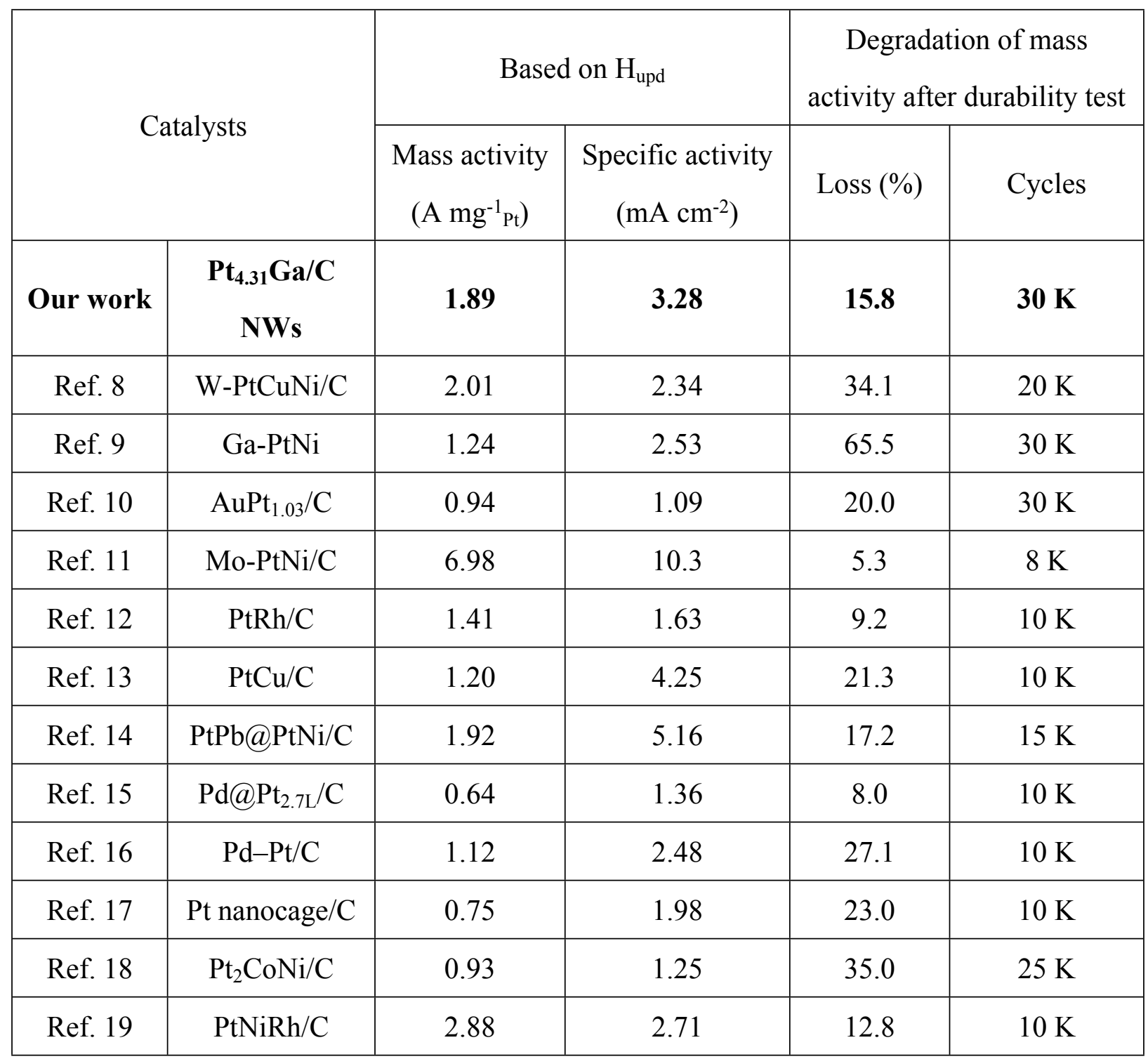




\section{References}

1. Becke, A. D.; Edgecombe, K. E. A Simple Measure of Electron Localization in Atomic and Molecular Systems. J. Chem. Phys. 1990, 92, 5397-5403.

2. Savin, A.; Nesper, R.; Wengert, S.; Fässler, T. F. ELF: The Electron Localization Function. Angew. Chem. Int. Ed. 1997, 36, 1808-1832.

3. Perdew, J. P.; Burke, K.; Ernzerhof, M. Generalized Gradient Approximation Made Simple. Phys. Rev. Lett. 1996, 77, 3865-3868.

4. Grimme, S.; Antony, J.; Ehrlich, S.; Krieg, S. A Consistent and Accurate Ab Initio Parametrization of Density Functional Dispersion Correction (DFT-D) for the 94 Elements H-Pu. J. Chem. Phys. 2010, 132, 154104.

5. Grimme, S.; Ehrlich, S.; Goerigk, L. Effect of the Damping Function in Dispersion Corrected Density Functional Theory. J. Comp. Chem. 2011, 32, 1456-1465.

6. Kresse, G.; Furthmüller, J. Efficient Iterative Schemes for Ab Initio Total-Energy Calculations Using a Plane-Wave Basis Set. Phys. Rev. B 1996, 54, 11169-11186.

7. Blöchl, P. E. Projector Augmented-Wave Method. Phys. Rev. B 1994, 50, 17953-17979.

8. Tu, W.; Chen, K.; Zhu, L.; Zai, H.; E, B.; Ke, X.; Chen, C.; Sui, M.; Chen, Q.; Li, Y. Tungsten-Doping-Induced Surface Reconstruction of Porous Ternary Pt-Based Alloy Electrocatalyst for Oxygen Reduction. Adv. Funct. Mater. 2019, 29, 1807070.

9. Lim, J.; Shin, H.; Kim, M.; Lee, H.; Lee, K. S.; Kwon, Y.; Song, D.; Oh, S.; Kim, H.; Cho, E. Ga-Doped Pt-Ni Octahedral Nanoparticles as a Highly Active and Durable Electrocatalyst for Oxygen Reduction Reaction. Nano Lett. 2018, 18, 2450-2458.

10. Bian, Ting.; Zhang, H.; Jiang, Y.; Jin, C.; Wu, J.; Yang, H.; Yang, D. Epitaxial Growth of Twinned Au-Pt Core-Shell Star-Shaped Decahedra as Highly Durable Electrocatalysts. Nano Lett. 2015, 15, 7808-7815.

11. Huang, X.; Zhao, Z.; Cao, L.; Chen, Y.; Zhu, E.; Lin, Z.; Li, M.; Yan, A.; Zettl, A.; Wang, Y. M.; Duan, X.; Huang, Y. High-Performance Transition Metal-Doped $\mathrm{Pt}_{3} \mathrm{Ni}$ Octahedra for Oxygen Reduction Reaction. Science 2015, 348, 1230-1234.

12. Huang, H.; Li, K.; Chen, Z.; Luo, L.; Gu, Y.; Zhang, D.; Ma, C.; Si, R.; Yang, J.; Peng, Z.; Zeng, J. Achieving Remarkable Activity and Durability toward Oxygen Reduction Reaction Based on Ultrathin Rh-Doped Pt Nanowires. J. Am. Chem. Soc. 2017, 139, 8152-8159.

13. Lu, B.; Sheng, T.; Tian, N.; Zhang, Z.; Xiao, C.; Cao, Z.; Ma, H.; Zhou, Z.; Sun, S. 
Octahedral PtCu Alloy Nanocrystals with High Performance for Oxygen Reduction Reaction and Their Enhanced Stability by Trace Au. Nano Energy 2017, 33, 65-71.

14. Bu, L.; Shao, Q.; E, B.; Guo, J.; Yao, J.; Huang, X. PtPb/PtNi Intermetallic Core/Atomic Layer Shell Octahedra for Efficient Oxygen Reduction Electrocatalysis. J. Am. Chem. Soc. 2017, 139, 9576-9582.

15. Wang, X.; Choi, S. I.; Roling, L. T.; Luo, M.; Ma, C.; Zhang, L.; Chi, M.; Liu, J.; Xie, Z.; Herron, J. A.; Mavrikakis, M.; Xia, Y. Palladium-Platinum Core-Shell Icosahedra with Substantially Enhanced Activity and Durability Towards Oxygen Reduction. Nat. Commun. 2015, 6, 7594.

16. He, D. P.; He, D.; Wang, J.; Lin, Y.; Yin, P.; Hong, X.; Wu, Y.; Li, Y. Ultrathin Icosahedral Pt-Enriched Nanocage with Excellent Oxygen Reduction Reaction Activity. J. Am. Chem. Soc. 2016, 138, 1494-1497.

17. Zhang, L.; Roling, L. T.; Wang, X.; Vara, M.; Chi, M.; Liu, J.; Choi, S. I.; Park, J.; Herron, J. A.; Xie, Z.; Mavrikakis, M.; Xia, Y. Platinum-Based Nanocages with Subnanometer-Thick Walls and Well-Defined, Controllable Facets. Science 2015, 349, 412-416.

18. Lokanathana, M.; Patila, I. M.; Navaneethand, M.; Pareya, V.; Thapaa, R.; Kakade, B. Designing of Stable and Highly Efficient Ordered $\mathrm{Pt}_{2} \mathrm{CoNi}$ Ternary Alloy Electrocatalyst: The Origin of Dioxygen Reduction Activity. Nano Energy 2018, 43, 219-227.

19. Li, K.; Li, X.; Huang, H.; Luo, L.; Li, X.; Yan, X.; Ma, C.; Si, R.; Yang, Zeng, J. One-Nanometer-Thick PtNiRh Trimetallic Nanowires with Enhanced Oxygen Reduction Electrocatalysis in Acid Media: Integrating Multiple Advantages into One Catalyst. J. Am. Chem. Soc. 2018, 140, 16159-16167. 